\title{
Attention to Bright Surfaces Enhances the Pupillary Light Reflex
}

\author{
Paola Binda, ${ }^{1,2}$ Maria Pereverzeva, ${ }^{1}$ and Scott 0. Murray $^{1}$ \\ ${ }^{1}$ University of Washington, Department of Psychology, Seattle, Washington 98195-1525, and ${ }^{2}$ Universita' di Pisa, Dipartimento Integrato Interistituzionale, \\ 56126 Pisa, Italy
}

One longstanding question is how early in the visual system attention exerts its influence. Here we show that an effect of attention can be measured at the earliest possible stage of visual information processing, as a change in the optics of the eye. We tested human subjects and found that covertly attending to bright surfaces results in an enhanced pupillary light reflex (PLR) — the pupillary constriction that occurs in response to light increments. The PLR optimizes the optical quality of the retinal image across illumination conditions, increasing sensitivity by modulating retinal illumination, and improving acuity by reducing spherical aberrations. The attentional modulation of the PLR that we describe constitutes a new mechanism through which vision is affected by attention; we discuss three alternatives for the neural substrates of this effect, including the possibility that attention might act indirectly, via its well established effects in early visual cortex.

\section{Introduction}

Selective attention is one of the primary means to optimize information processing. It has been suggested to act through multiple mechanisms, including enhancement of sensory signal-to-noise ratios by increasing gain and response reliability (McAdams and Maunsell, 1999; Reynolds et al., 2000) and dynamically adjusting receptive field properties such as their size (Desimone and Duncan, 1995; Connor et al., 1997) and position (Womelsdorf et al., 2006) to meet the specific behavioral demands of the observer (Carrasco, 2011). These mechanisms appear to be implemented at early stages of visual processing - for instance, by selectively increasing spike rate as early as in the thalamus (McAlonan et al., 2008), and by narrowing the tuning for spatial position in V1 neural populations (Fischer and Whitney, 2009).

However, even before visual information enters the neural processing stream, its quality is determined by retinal and optical factors. In particular, the diameter of the pupil has been shown to affect visual signals in multiple ways. At very low light levels, dilated pupils increase the probability of photon capture by the retina, increasing sensitivity. At high light levels, pupil constriction reduces the level of light adaptation, thereby reducing the time required to restore sensitivity after an abrupt light decrement (Woodhouse and Campbell, 1975). In less extreme conditions, pupil size affects acuity; smaller pupils reduce spherical aberrations and enhance the optical quality of the retinal image.

\footnotetext{
Received July 14, 2012; revised Nov. 29, 2012; accepted Dec. 7, 2012.

Author contributions: P.B., M.P., and S.O.M. designed research; P.B., M.P., and S.O.M. performed research; P.B. and S.O.M. analyzed data; P.B. and S.O.M. wrote the paper.

This work was supported by the EC FP7 Marie Curie IOF fellowship AWESOMe to P.B. and funding from the University of Washington Royalty Research Fund to S.O.M.

The authors declare no competing financial interests.

Correspondence should be addressed to Dr. Paola Binda, University of Washington, Department of Psychology, Guthrie Hall, Box351525, Seattle,WA 98195-1525. E-mail: pbinda1@uw.edu or p.binda1@in.cnr.it.

DOI:10.1523/JNEUROSCI.3440-12.2013

Copyright $\odot 2013$ the authors $\quad 0270-6474 / 13 / 332199-06 \$ 15.00 / 0$
}

The pupillary light reflex (PLR) ensures that pupil diameter remains optimal across a wide range of luminance levels (Campbell and Gregory, 1960). Here we ask whether attention exerts its control over this simple low-level behavior, affecting pupil size and thereby the quality of visual information at the earliest possible stage of visual processing, the optics of the eye.

\section{Materials and Methods}

Subjects and apparatus. A total of 10 subjects (3 authors, 4 females) gave informed consent to participate in one or more of the experiments reported here, which were approved by the University of Washington $\mathrm{Hu}$ man Subjects Institutional Review Board.

Subjects viewed a $35 \times 28 \mathrm{~cm}$ calibrated CRT monitor from a distance of $81 \mathrm{~cm}$; a chin rest was used to stabilize head position. The experimental room had no illumination other than the display screen. Displays were generated in Matlab (Mathworks) using the Psychophysics Toolbox (Brainard, 1997). Stimuli were presented against a uniform gray background $\left(50 \mathrm{~cd} / \mathrm{m}^{2}\right)$. Subjects were asked to refrain from blinking at all times except during the intertrial interval (defined in Stimuli and task, below) and to maintain their gaze on a fixation mark at the center of the screen (except when otherwise stated).

Stimuli and task. In Experiment 1 (4 subjects), the display consisted of two disks, $7^{\circ}$ in diameter, centered at $8^{\circ}$ eccentricity left or right of fixation, one bright and the other dark (both with $90 \%$ contrast relative to the gray background). The disks were presented $2 \mathrm{~s}$ after the trial onset, lasted $6 \mathrm{~s}$, and were followed by an intertrial interval of $2 \mathrm{~s}$ (yielding a $10 \mathrm{~s}$ trial duration). Three conditions were tested.

In the Attention condition, the presentation of the disks was preceded by a central cue (a line extending left or right from the fixation mark, presented at trial onset and visible until stimulus offset), which instructed subjects to attend to the left or right stimulus. Attention direction and spatial position of the bright and dark disks were counterbalanced across trials. To ensure task engagement, subjects counted, and reported with a button press during the intertrial-interval, the number (1-3) of brief $(200 \mathrm{~ms})$ color changes of a small dot located in the center of the attended disk, while ignoring color changes in the dot located on the unattended disk. Dots changed from cyan to a greenish color 
and the percentage of blue light in the greenish target could be adjusted to maintain performance around the $70 \%$ level - the proportion of correct responses when the bright or the dark disk was attended was 0.68 or 0.72 , respectively (twotailed paired $t$ test, $t=-1.06, p>0.3)$.

In the other two conditions, the stimuli, procedures, and task were the same except for the following. In the Direct Fixation condition, the central cue instructed subjects to shift their gaze to the dot marking the center of the left or right disk; this eccentric gaze position was acquired before the onset of the disks and it was maintained throughout their duration. In the Control experiment, no central cue was presented; subjects maintained attention on the fixation mark, which was displaced left or right by an amount matching the maximum gaze displacement measured for the same subject in the Attention condition (the 95th percentile of the eye position bias in the direction of the attended stimulus, see below). The fixation mark was displaced at the beginning of the trial and returned to the center after the disks were extinguished. Subjects counted color changes of the fixation mark; no color change occurred in the dots at the center of the disks.

Experiment 2 (6 subjects) was performed with identical procedures as Experiment 1 but with different stimuli and task details. The stimuli were square-wave $1 \mathrm{cpd}$ luminance decrements or increments $(90 \%$ increment/ decrement, inscribed in the same area covered by the disks of Experiment 1; the bright and dark gratings were identical in all respects but luminance). Subjects counted the number (1-3) of small, rapid rotations $\left( \pm 0.25^{\circ}\right.$, identical for bright and dark) of the attended stimulus, while ignoring the rotations of the unattended stimulus. In the Attention condition, task performance was slightly worse for the bright than for the dark stimulus: proportion correct was 0.69 versus 0.76 , respectively (two-tailed pairedsample $t$ test, $t=5.59, p<0.01)$.

Experiment 3 ( 4 subjects) tested a variation of the Attention condition of Experiment 1, where the attention cue was delayed relative to the disks presentation (i.e., relative to Experiment 1, the order of the two events was inverted). The disks were visible for a total of $8 \mathrm{~s}$, from trial onset to the intertrial interval; the cue was presented $2 \mathrm{~s}$ into the trial and remained visible until stimulus offset.

Finally, Experiment 4 measured the effect of stimulus intensity on attention-induced pupil size changes. Stimuli, task, and procedures were the same as in the Attention condition of Experiment 1, except for the following. In the first condition ( 2 subjects), the intensity of both disks was simultaneously varied $(90 \%, 45 \%$, and $22.5 \%$ of the maximum attainable contrast). In the second condition (4 subjects), only one disk (bright or dark) was presented at maximum intensity. The position of the disk was constant within a session and counterbalanced across sessions (to control for the possible confounding effect of afterimages).

Eye tracking. Pupil diameter and 2D eye position were measured monocularly with a video-based eye-tracker (ASL Eyetrack 6, with a remote sensor mounted below the monitor screen). A standard 9 point calibration was run at the beginning of each session. Eye tracking data were acquired at $120 \mathrm{~Hz}$. Time points with unrealistic pupil size $(<2 \mathrm{~mm}$ or $>8 \mathrm{~mm}$ ) or eye position (locations outside the screen monitor) were treated as signal losses. Traces were averaged in $250 \mathrm{~ms}$ temporal bins. Trials where horizontal eye position in any temporal bin deviated by $>2^{\circ}$ from the fixation point were eliminated $(<1 \%)$. An additional analysis further excluded all trials where raw eye position (i.e., even a single sam- ple acquired at $120 \mathrm{~Hz}$ ) deviated by $>2^{\circ}$ from fixation $(7 \%)$. For all experiments, analyses included a minimum of 50 trials per subject and condition (attend to/fixate on/bias eye position toward: the brighter or darker region).

For each subject and condition, pupil data were averaged across trials, after subtracting pupil diameter in the $500 \mathrm{~ms}$ preceding stimulus presentation. These traces were averaged across subjects yielding the gray and black curves in Figures 1, 2B-D , and 3B. Also, the difference between the two traces was computed for each subject and then averaged across subjects to give the blue curve in the same figures. The statistical significance of the effects in the Attention and Control conditions of Experiments 1 and 2 was assessed as follows: the average difference between traces was computed over the time of stimulus presentation (between 2 and $8 \mathrm{~s}$ from the beginning of the trial), normalized to the same value observed in the Direct Fixation experiment (so to minimize intersubject variability), and submitted to a two-tailed one-sample $t$ tests (null hypothesis: 0 pupil size difference across conditions).

Horizontal eye position data were analyzed in the same way as pupil size traces, except trials were divided in four categories according to the luminance (bright/dark) and position (left/right) of the attended disk. In addition, we examined the distribution of the horizontal eye position bias toward the attended stimulus. Individual data samples (not averaged in temporal bins) acquired during the stimulus presentation window (2-8 s) were considered. The 95th percentile of the distribution was taken as an estimate of the largest eye position bias, at any time, toward 

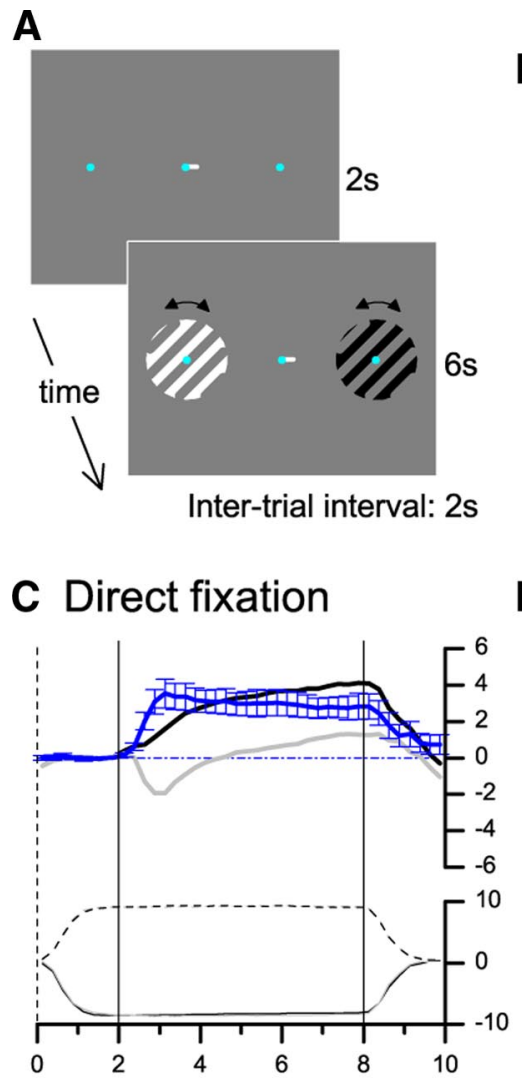

B

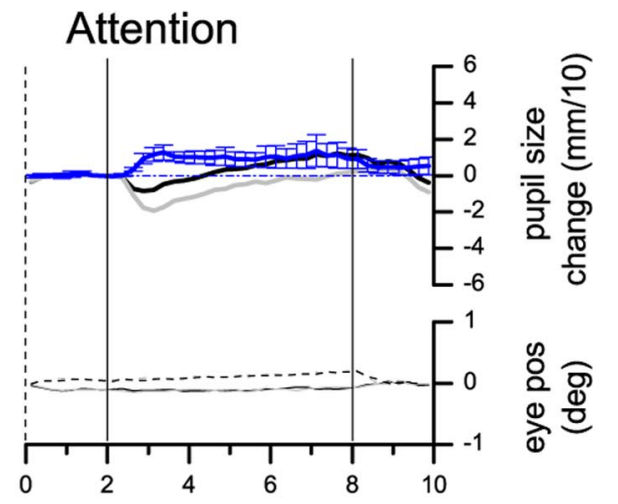

D Control

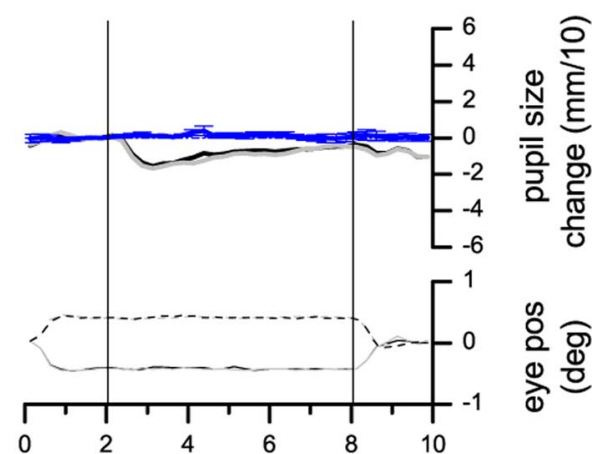

time from trial onset (sec)

Figure 2. Experiment 2: Effect of covertly attending to or directly fixating bright and dark gratings. $\boldsymbol{A}-\boldsymbol{D}$ follow the same conventions as in Figure 1.
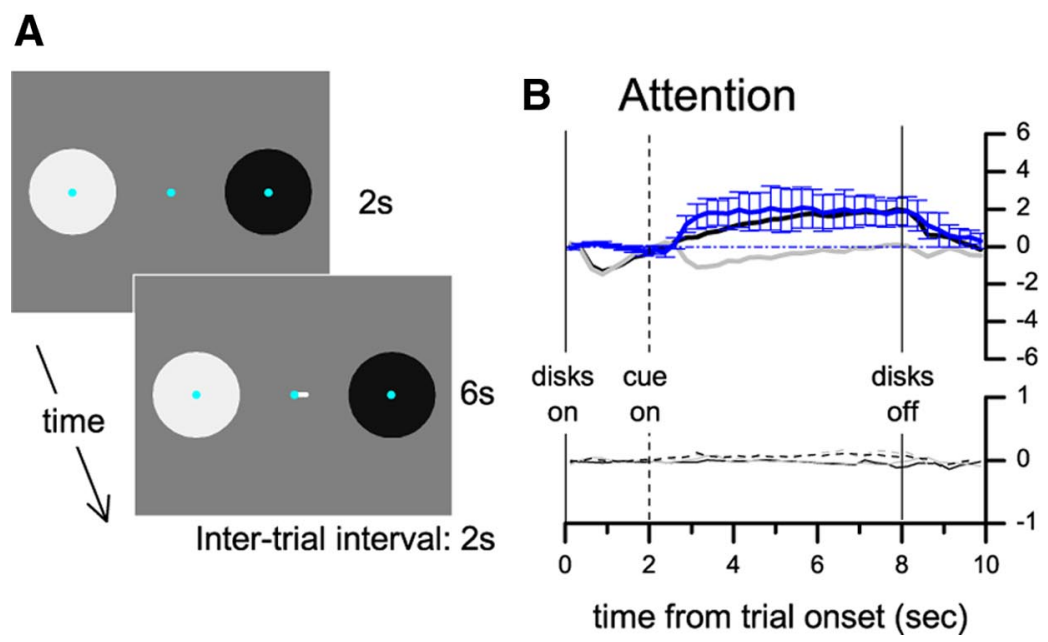

Figure 3. Experiment 3: Effect of covert attention to dark and bright disks in a delayed cue paradigm. $\boldsymbol{A}$, Stimulus display. $\boldsymbol{B}$, Same conventions as in Figure 1.

the attended stimulus. This value was computed for each subject in the Attention condition of Experiments 1 and 2 and used in the Control condition of the same experiments, as described above.

\section{Results}

Experiment 1 measured pupil size while subjects fixated the center of a uniform gray screen and two disks, one bright and one dark, were presented at equal eccentricity in the left and right periphery. Preceding the onset of the disks, a central cue in-

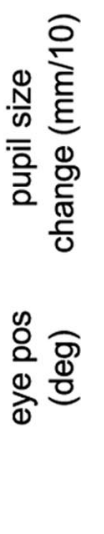

structed subjects to covertly shift attention to the left or the right disk (Fig. 1A). Subjects reported the number of brief chromaticity changes of a small dot located in the center of the attended disk, while ignoring color changes on the unattended side (changes were adjusted to maintain performance at $\sim 70 \%$, equal across conditions). The stimulus and the task were identical across trials. However, the diameter of the pupil was systematically smaller when the bright disk was attended relative to when the dark disk was attended (Fig. 1B, light gray and dark curves, respectively; the blue curve shows the average pupil size difference between conditions).

We compared the size of this effect to the change in pupil size induced by directly fixating the bright or the dark disk. This was measured using the same experimental procedures except that the central cue instructed subjects to move their eyes to the center of the left or right disk (Fig. $1 C)$. The pupil size difference induced by covertly attending to the bright versus dark disk was $37 \%$ of the effect induced by shifting gaze to directly fixate either region (two-tailed $t$ test, $t=4.84, p<0.05$ ).

In general, subjects were very good at maintaining central fixation. Deviations in the direction of the attended side were close to the resolution limit of our eyetracking system $\left(0.5^{\circ}\right.$ by manufacturer's specifications), resulting in average eye position indistinguishable across conditions (Fig. $1 B$, eye position traces). However, in a control experiment, we further excluded the possibility that small deviations of eye position toward the attended disk could explain the observed effect. Subjects maintained their attention on the fixation mark to detect brief color changes. Gaze direction was biased toward one of the disks by displacing the fixation mark. The amount of displacement matched the largest (95th percentile) gaze shifts observed for each subject in the Attention condition $\left(\sim 0.5^{\circ}\right)$, but no difference in pupil size as a function of gaze direction was observed (Fig. $1 D$, pupil size difference was 0.08 of the effect in the Direct Fixation condition, two-tailed $t$ test, $t=0.99, p>0.3$ ).

We replicated these results in Experiment 2, where the details of the behavioral task and the stimulus layout were different (Fig. 2A). The stimuli were square-wave 1 cpd luminance decrements or increments. Subjects reported the number of small, rapid rotations $\left( \pm 0.25^{\circ}\right.$, identical for bright and dark) of the attended stimulus, while ignoring the rotations of the unattended stimulus. Pupil diameter was smaller when attending to the bright stimulus compared with the dark stimulus (Fig. 2B); this difference was 31\% (two-tailed $t$ test, $t=3.62, p<0.01$ ) of 
that evoked by directly looking at the bright or the dark stimulus (Fig. 2C) and small gaze shifts toward the bright or the dark stimulus, when both were unattended, did not produce a differential pupillary response (Fig. 2D, pupil size difference was 0.03 of the effect in the Direct Fixation condition, two-tailed $t$ test, $t=0.94, p>0.3$ ).

The results of the Control condition in Experiments 1 and 2 show that small and steady eye position biases cannot account for the differences in pupil size in the Attention condition. We also excluded the possibility that brief but large gaze shifts toward the attended stimulus systematically affected pupil size (these transients might go undetected in the binned data). To this end, we eliminated all trials where the horizontal deviation of gaze exceeded $2^{\circ}$ even in a single $\approx 8 \mathrm{~ms}$ long sample (a minority of our dataset, $7 \%$ ) and verified that the results were not affected (pupil size change in the Attention condition was $36 \%$ and $29 \%$ of the effect in the Direct Fixation condition for Experiments 1 and 2 respectively, statistically significant at $p<0.05$ in both cases).

In Experiments 1 and 2, the spaceaveraged luminance of the display was always constant, yet the onset of the disks evoked a transient pupillary constriction (visible in all conditions; Fig. $1 B-D$ ). This is consistent with previous studies showing that a transient response can be evoked by several non-luminance-modulated stimuli (Barbur, 2004). A modification of our paradigm allowed us to dissociate this response from the attention-induced luminance-dependent pupillary response. In Experiment 3, stimuli and task were the same as in Experiment 1, but the disks were presented first, followed by the attentional cue after a $2 \mathrm{~s}$ delay period. Figure 3 shows that the initial constriction caused by the appearance of the disks is virtually identical across trials; a relative pupillary constriction starts only $\sim 1 \mathrm{~s}$ after the cue instructed subjects to pay attention to the bright disk, and it is maintained until the cue and the stimulus disappear.

The PLR is more pronounced as stimulus luminance increases (Clarke et al., 2003); Experiment 4 examined whether the same holds true for the attention-induced pupillary constriction. We repeated Experiment 1 with stimuli of different intensity, 1/2 and $1 / 4$ of the original luminance increments and decrements- the contrast of both the bright and the dark disk was $90 \%, 45 \%$, and $22 \%$. Figure $4, A$ and $B$, shows pupil size traces for the three stimulus intensities, separately for trials where the bright and the dark disk were attended. When the bright disk was attended (Fig. $4 A$ ), increased stimulus luminance led to more pronounced pupillary constriction. Figure $4 B$ shows that, in trials where the dark disk was attended, there was no clear dependency of pupil size on the magnitude of the luminance decrement, indicating an asymmetric effect of attending to bright and dark surfaces. We investigated this aspect further by measuring attention-modulated pupillary responses to net increments or decrements of light.
Only one disk was presented, either bright or dark, preceded by the cue to direct attention to the disk or to the uniform gray background at the opposite side of fixation (Fig. $4 C, D$ ). The presentation of the bright disk induced pupillary constriction (the pupillary light reflex), and this was enhanced when attention was directed to the stimulus. However, when the dark disk was presented, pupil size was independent of the direction of attention.

\section{Discussion}

In four experiments, we showed that covertly attending to brighter image regions induces changes in pupil size that are $\sim 30 \%$ of those induced by shifting gaze to directly look at the stimuli (Experiments 1 and 2), that pupil size changes can be elicited by shifting attention across an unchanged visual image (Experiment 3), and that the attentional modulation of pupil size is dependent on stimulus strength and is only associated with luminance increments and not luminance decrements (Experiment 4). In all experiments, we enforced fixation; small eye movements (below the resolution of our eye tracking apparatus, e.g., microsaccades) could not account for the reported effects of attention, since in control conditions we showed that large and systematic eye position biases did not affect pupil size when attention was directed away from the stimuli.

Factors other than light level are known to affect pupil size, and these effects can be detected in our results. Pupillary constric- 
tion accompanies focal adjustment (Marg and Morgan, 1949) and can be transiently evoked by a variety of non-luminancemodulated stimuli (Barbur, 2004); the short-lived constriction we observed following the onset of the two disks (most evident in Figs. $1 D, 2 D$, and $3 B$ ) may be an example of the latter phenomenon. Nonvisual factors such as cognitive effort, memory load, and perceptual switches are known to induce pupil dilation (Kahneman and Beatty, 1966; Aston-Jones and Cohen, 2005; Einhäuser et al., 2008), possibly as a by-product of variations in sympathetic activity. The progressive pupil dilation across the duration of each trial observed in all our experiments can be explained, at least in part, by the cognitive effort associated with performing our psychophysical task. Importantly, while nonluminance-related factors likely affected our recordings, they cannot explain the main effect we report: a differential pupil response depending on the luminance at the attended location, observed with identical stimulus and task.

The asymmetry of the attentional effect revealed by Experiment 4 - that pupillary constriction, but not pupillary dilation, is modulated by attention-is compatible with different hypotheses. The dilation of the pupils in response to a light decrement is controlled by a neural circuit separate from the one mediating pupillary constriction, described below (Loewenfeld, 1993). One possibility is that attention selectively modulates activity in one but not the other circuit. Alternatively, it is possible that the pupil dilation (relative to prestimulus baseline) observed in our experiment is primarily explained by the effect of task engagement and cognitive effort (Kahneman and Beatty, 1966), and independent from pupillary light responses and their attentional modulation. Further research is needed to distinguish between these possibilities.

Our experiments show that attention to a bright surface can induce pupillary constriction and it is well established that pupillary constriction is the result of increased output of the mesencephalic Edinger-Wesphal (EW) nucleus (Loewenfeld, 1993). The primary circuit responsible for the pupillary constriction in response to light (PLR) is subcortical, with the EW nucleus receiving excitatory input from the pretectal olivary nucleus $(\mathrm{PON})$, in turn activated by retinal signals [mainly the melanopsin-containing retinal ganglion cells (Güler et al., 2008)]. However, there are three known modulatory inputs to this circuit. First, there are anatomical projections from the visual cortex and from the superior colliculus to the PON (Gamlin, 2006), which could enhance its response to light and thereby increase EW activity. Second, the EW has been suggested to receive excitatory input from extrastriate visual areas (Loewenfeld, 1993). Third, the EW receives inhibitory input from the sympathetic system, including projections from the locus ceruleus and the hypothalamus (Koss et al., 1984) that are potentially under cortical control (Aston-Jones and Cohen, 2005), and a reduction in this inhibitory input can result in pupil constriction (Wilhelm et al., 2002).

The attentional enhancement of the pupillary response to light we report bears a strong resemblance to the well established attention-induced enhancement of neural responses in early visual cortex (Reynolds et al., 2000; Martínez-Trujillo and Treue, 2002; Reynolds and Chelazzi, 2004; Khayat et al., 2010). This analogy may favor the hypothesis that the attentional modulation of the PLR depends on early visual cortex signals feeding back into the PLR circuit and reflecting an evaluation of the behaviorally relevant light level in the visual scene-a possibility that is consistent with previous reports demonstrating cortical modulations of the PLR (Lorber et al.,
1965; Barbur, 2004). However, our results remain compatible with multiple neural pathways that require further research to identify. Another open question concerns the spatial specificity of the effect. It has been shown that the effects of attention on neural responses can have sharp spatial selectivity (Connor et al., 1997). In our experiments, attended and unattended stimuli were always located in opposite hemifields. Thus, further experiments are required to establish the spatial selectivity of the attentional modulation of the PLR.

Attention has been proposed to enhance visual sensitivity and acuity by boosting neural responses and modifying the receptive field structure of neurons at the earliest stages of processing (Reynolds and Chelazzi, 2004; Womelsdorf et al., 2006; Carrasco, 2011). Our results indicate that attention can also affect the input to these processes by modulating pupil size, and thereby enhancing the optical quality of the retinal image. Pupil size changes on the order of $1 \mathrm{~mm}$ have a dramatic effect on sensitivity to spatial frequencies close to the acuity limit, modulating it by up to $1 \log$ unit (Campbell and Gregory, 1960). The magnitude of attentioninduced pupil size changes observed in our experiments is small, on the order of $0.2 \mathrm{~mm}$. However, the dependence on stimulus intensity revealed by Experiment 4 implies that much more prominent changes should occur in natural settings, where luminance variations are several orders of magnitude larger than those attainable within our experimental set up (luminance ranged between 5 and $105 \mathrm{~cd} / \mathrm{m}^{2}$ ).

In conclusion, our findings reveal a previously undocumented mechanism through which attention affects visual processing. Regardless of which specific neural pathway is involved, our experiments show that attention modifies the optical quality of the retinal image, thereby exerting its influence even at the point where the light enters the eye: the pupil.

\section{References}

Aston-Jones G, Cohen JD (2005) An integrative theory of locus coeruleusnorepinephrine function: adaptive gain and optimal performance. Annu Rev Neurosci 28:403-450. CrossRef Medline

Barbur J (2004) Learning from the pupil: studies of basic mechanisms and clinical applications. In: The visual neurosciences (Chalupa L, Werner J, eds), pp 641-656. Cambridge: MIT.

Brainard DH (1997) The psychophysics toolbox. Spat Vis 10:433-436. CrossRef Medline

Campbell FW, Gregory AH (1960) Effect of size of pupil on visual acuity. Nature 187:1121-1123. CrossRef Medline

Carrasco M (2011) Visual attention: the past 25 years. Vision Res 51:14841525. CrossRef Medline

Clarke RJ, Zhang H, Gamlin PD (2003) Characteristics of the pupillary light reflex in the alert rhesus monkey. J Neurophysiol 89:3179-3189. CrossRef Medline

Connor CE, Preddie DC, Gallant JL, Van Essen DC (1997) Spatial attention effects in macaque area V4. J Neurosci 17:3201-3214. Medline

Desimone R, Duncan J (1995) Neural mechanisms of selective visual attention. Annu Rev Neurosci 18:193-222. CrossRef Medline

Einhäuser W, Stout J, Koch C, Carter O (2008) Pupil dilation reflects perceptual selection and predicts subsequent stability in perceptual rivalry. Proc Natl Acad Sci U S A 105:1704-1709. CrossRef Medline

Fischer J, Whitney D (2009) Attention narrows position tuning of population responses in V1. Curr Biol 19:1356-1361. CrossRef Medline

Gamlin PD (2006) The pretectum: connections and oculomotor-related roles. Prog Brain Res 151:379-405. CrossRef Medline

Güler AD, Ecker JL, Lall GS, Haq S, Altimus CM, Liao HW, Barnard AR, Cahill H, Badea TC, Zhao H, Hankins MW, Berson DM, Lucas RJ, Yau KW, Hattar S (2008) Melanopsin cells are the principal conduits for rod-cone input to non-image-forming vision. Nature 453:102-105. CrossRef Medline 
Kahneman D, Beatty J (1966) Pupil diameter and load on memory. Science 154:1583-1585. CrossRef Medline

Khayat PS, Niebergall R, Martinez-Trujillo JC (2010) Attention differentially modulates similar neuronal responses evoked by varying contrast and direction stimuli in area MT. J Neurosci 30:2188-2197. CrossRef Medline

Koss MC, Gherezghiher T, Nomura A (1984) CNS adrenergic inhibition of parasympathetic oculomotor tone. J Auton Nerv Syst 10:55-68. CrossRef Medline

Loewenfeld I (1993) The pupil: anatomy, physiology, and clinical applications. Detroit: Wayne State UP.

Lorber M, Zuber BL, Stark L (1965) Suppression of pupillary light reflex in binocular rivalry and saccadic suppression. Nature 208:558. CrossRef

Marg E, Morgan MW Jr (1949) The pupillary near reflex; the relation of pupillary diameter to accommodation and the various components of convergence. Am J Optom Arch Am Acad Optom 26:183-198. Medline

Martínez-Trujillo J, Treue S (2002) Attentional modulation strength in cortical area MT depends on stimulus contrast. Neuron 35:365-370. CrossRef Medline
McAdams CJ, Maunsell JH (1999) Effects of attention on the reliability of individual neurons in monkey visual cortex. Neuron 23:765773. CrossRef Medline

McAlonan K, Cavanaugh J, Wurtz RH (2008) Guarding the gateway to cortex with attention in visual thalamus. Nature 456:391-394. CrossRef Medline

Reynolds JH, Chelazzi L (2004) Attentional modulation of visual processing. Annu Rev Neurosci 27:611-647. CrossRef Medline

Reynolds JH, Pasternak T, Desimone R (2000) Attention increases sensitivity of V4 neurons. Neuron 26:703-714. CrossRef Medline

Wilhelm BJ, Wilhelm H, Moro S, Barbur JL (2002) Pupil response components: studies in patients with Parinaud's syndrome. Brain 125: 2296-2307. CrossRef Medline

Womelsdorf T, Anton-Erxleben K, Pieper F, Treue S (2006) Dynamic shifts of visual receptive fields in cortical area MT by spatial attention. Nat Neurosci 9:1156-1160. CrossRef Medline

Woodhouse JM, Campbell FW (1975) The role of the pupil light reflex in aiding adaptation to the dark. Vision Res 15:649-653. CrossRef Medline 